\title{
GAMMA-GAMMA DIRECTIONAL CORRELATION MEASUREMENTS IN THE DECAY OF Ba ${ }^{133}$
}

\author{
L. I. YIN and M. L. WIEDENBECK \\ Harrison M. Randall Laboratory of Physics, \\ The University of Michigan, \\ Ann Arbor, Michigan ${ }^{\dagger}$
}

Received 13 January 1964

Abstract: The gamma rays following the electron capture decay of $\mathrm{Ba}^{138}$ have been studied using coincidence and directional correlation techniques. The existence of gamma rays with energies of $162 \mathrm{keV}$ and $222 \mathrm{keV}$ has been ascertained. The correlation functions for the $356 \mathrm{keV}-82$ $\mathrm{keV}, 302 \mathrm{keV}-82 \mathrm{keV}, 80 \mathrm{keV}-82 \mathrm{keV}$ and $276 \mathrm{keV}-162 \mathrm{keV}$ cascades are found to be, respectively: $W(\theta)=1+(0.0331 \pm 0.0017) P_{8}(\cos \theta)+(0.0045 \pm 0.0033) P_{4}(\cos \theta)$, $W(\theta)=1-(0.0238 \pm 0.0060) P_{2}(\cos \theta)+(0.0061 \pm 0.0089) P_{4}(\cos \theta)$, $W(\theta)=1+(0.0319) \pm 0.0045) P_{2}(\cos \theta)+(0.005 \pm 0.009) P_{d}(\cos \theta)$, $W(\theta)=1-(0.328 \pm 0.009) P_{2}(\cos \theta)-(0.067 \pm 0.016) P_{4}(\cos \theta)$.

These results support the spin sequence of $\frac{7^{+}}{2}, \frac{5}{2}+\frac{5}{2}+\frac{3^{+}}{2}$ and $\frac{1^{+}}{2}$, for the ground state, 82, 162, 384 and $438 \mathrm{keV}$ levels of $\mathrm{Cs}^{133}$. The measured mixing ratios of the following gamma rays are: $80 \mathrm{keV}:(63 \pm 9) \%$ E2 $+(37 \pm 9) \% \mathrm{M} 1 ; 82 \mathrm{keV}:(97.6 \pm 0.1) \% \mathrm{M} 1+(2.4 \pm 0.1) \% \mathrm{E} 2 ; 162 \mathrm{keV}:$ $(95.1 \pm 0.6) \% \mathrm{E} 2+(4.9 \pm 0.6) \% \mathrm{M} 1 ; 276 \mathrm{keV}:$ pure E2; $302 \mathrm{keV}:(99.7 \pm 0.1) \% \mathrm{M} 1+(0.3 \pm 0.1) \%$ E2; $356 \mathrm{keV}$ : pure E2.

RADIOACTIVITY Ba $^{138}$ [from Oak Ridge];

measured $\gamma \gamma$-coin, $\gamma \gamma(\theta) . \mathrm{Cs}^{138}$ deduced levels, $J, \pi, \delta(\mathrm{E} 2 / \mathrm{M} 1)$.

\section{Introduction}

The nuclear levels of $\mathrm{Cs}^{133}$ have been studied by many authors ${ }^{1-13}$ ). Most of the recent investigators $7,8,14$ ) are in agreement concerning the salient features of the electron capture decay scheme of $7.2 \mathrm{y} \mathrm{Ba}^{133}$ as shown in fig. 1. Excited levels of $\mathrm{Cs}^{133}$ at $82,162,384$ and $438 \mathrm{keV}$ have been established. The majority of authors are also in accord concerning the existence of all gamma ray transitions shown in fig. 1, except the two of energies $162 \mathrm{keV}$ and $222 \mathrm{keV}$. These two gamma rays have been observed by Stewart and $\mathrm{Lu}^{8}$ ), but not by Ramaswamy et al. ${ }^{11}$ ). Through the measurement of relative gamma ray intensities, Stewart and $\mathrm{Lu}^{8}$ ) also deduced an additional electron capture transition from the ground state of $\mathrm{Ba}^{133}$ leading directly to the $162 \mathrm{keV}$ level; whereas other authors observed only the two transitions leading to the 384 and the $438 \mathrm{keV}$ levels. Although the spins and parities of the ground state, 82,384 and $438 \mathrm{keV}$ levels of $\mathrm{Cs}^{133}$ are fairly certain to be $\frac{7}{2}^{+}, \frac{5}{2}^{+}, \frac{3}{2}^{+}$and $\frac{1}{2}^{+}$respectively, the spin of the $162 \mathrm{keV}$ level has been a point of dissent among authors. Bodenstedt et al. ${ }^{7}$ ) determined the spin of this level to be $\frac{5}{2}$ through directional

† Work supported in part by the U.S. Atomic Energy Commission. 
correlation measurement of the $276 \mathrm{keV}-162 \mathrm{keV}$ cascade; although they could not determine the mixing ratio of the $162 \mathrm{keV}$ gamma ray from their results. Ramaswamy et al. ${ }^{11,12}$ ) also determined the spin of this level to be $\frac{5}{2}$ based on the absence of an electron capture transition leading to this level from the ground state of $\mathrm{Ba}^{133}$ $\left(\frac{1}{2}^{+}\right)$. However, since Stewart and $\mathrm{Lu}^{8}$ ) did find an electron capture transition leading to this level, their interpretation of this branch as an allowed transition $(\log f t=9)$ requires the spin to be $\frac{3}{2}$. Recently, the directional correlation measurement of Arya ${ }^{13}$ ) on the $80 \mathrm{keV}-82 \mathrm{keV}$ cascade also assigns a spin of $\frac{3}{2}$ to this level. It is the purpose of the present investigation to resolve some of these uncertainties.

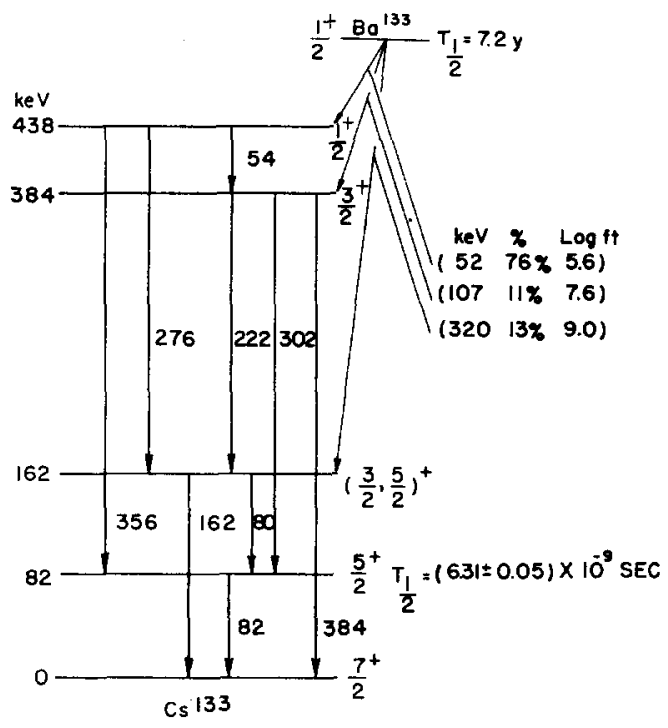

Fig. 1. Decay scheme of $\mathrm{Ba}^{138}$.

\section{Experimental Procedure}

$\mathrm{Ba}^{133}$ in the form of $\mathrm{HCl}$ solution was obtained from Oak Ridge. The liquid source, upon dilution, was injected into a thin-walled cylindrical lucite container of $4 \mathrm{~mm}$ diameter for both coincidence and directional correlation measurements. Two $5.1 \mathrm{~cm}$ by $5.1 \mathrm{~cm} \mathrm{NaI}(\mathrm{Tl})$ crystals mounted on RCA 6342A phototubes were used as detectors, with lateral lead shieldings to eliminate counter-to-counter scatterings. A fast-slow coincidence circuit with a resolving time of about 40 ns was employed in all cases; and energy selection was provided by two differential analysers. For coincidence measurements, the pulses coincident with the selected energy range were fed through a linear gate to be recorded on a 256-channel analyser. For each coincidence measurement, a corresponding accidental coincidence measurement for the same duration was also taken and subtracted in order to obtain the true coin- 
cidence spectrum. In all directional correlation measurements, the source to detector distance was $7 \mathrm{~cm}$; and data were taken at every $15^{\circ}$ in a double-quadrant sequence. The instruments were calibrated after each run to insure their stability. The true coincidence rate was furthermore divided by the product of the single counting rates to correct for possible electronic drifts. After making a least-square fit of the correlation data ${ }^{15}$ ), the expansion coefficients were normalized and corrected for finite angular resolution ${ }^{16}$ ).

\section{Coincidence Results}

As a preliminary preparation for directional correlation measurements, a series of coincidence spectra was collected. All results confirm the decay scheme of fig. 1 . A few of the coincidence spectra of special interest will be presented here.

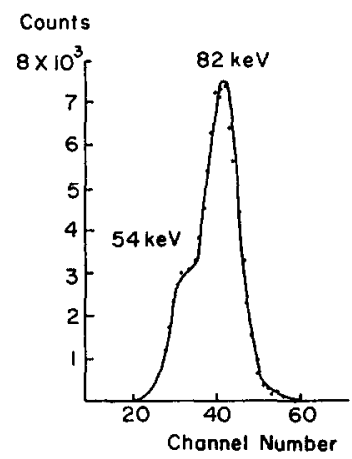

Fig. 2. Spectrum of gamma rays in coincidence with energies greater than $374 \mathrm{keV}$.

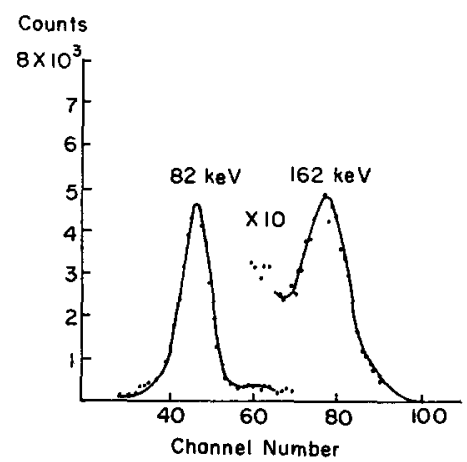

Fig. 3. Spectrum of gamma rays in coincidence with the energy range 264 to $284 \mathrm{keV}$.

Fig. 2 shows the spectrum of gamma rays which are coincident with energies greater than $374 \mathrm{keV}$. Since the selecting discriminator inevitably accepts some portion of the $356 \mathrm{keV}$ photopeak, one notices a rather pronounced $82 \mathrm{keV}$ peak in the coincidence spectrum. However, it is also clear that there is an enhancement at $54 \mathrm{keV}$ which is much more pronounced than the normal escape peak of the $82 \mathrm{keV}$ gamma ray. The existence of this gamma ray at $54 \mathrm{keV}$ in coincidence with energies higher than $374 \mathrm{keV}$ lends support to the decay scheme of fig. 1.

Fig. 3 shows the spectrum of gamma rays in coincidence with the energy range of 264 to $284 \mathrm{keV}$. The peaks at $80 \mathrm{keV}$ and $162 \mathrm{keV}$ are clearly evident. This spectrum demonstrates not only the existence of the $162 \mathrm{keV}$ gamma ray, but also its coincidence relationship with the $276 \mathrm{keV}$ gamma ray. Since the selected energy range also includes the Compton continuum of higher energy gamma rays, a portion of the area underneath the $80 \mathrm{keV}$ peak is due to the contribution of the $82 \mathrm{keV}$ gamma ray which is coincident with these high energy gamma rays as well as with the 276 $\mathrm{keV}$ itself. 
The coincidence spectrum of the energy range, 148 to $172 \mathrm{keV}$, is shown in fig. 4 . The $276 \mathrm{keV}$ gamma ray, whose intensity is so low that its photopeak is almost completely masked by the 356 and $302 \mathrm{keV}$ gamma rays in the single spectrum, now stands out very prominently. Also noteworthy is the weak, but definite peak at 222 $\mathrm{keV}$. The existence of this gamma ray was previously considered questionable. The peak at $162 \mathrm{keV}$ is coincident with the Compton continuum of the $276 \mathrm{keV}$ gamma ray, which is also being accepted in the energy selector.

The single spectrum of $\mathrm{Ba}^{133}$ as well as other coincidence spectra are well known in the literature ${ }^{1-13}$ ). Therefore, in order to avoid repetition, they will not be presented here. In general, it can be said that all coincidence measurements completely supports the decay scheme of fig. 1.

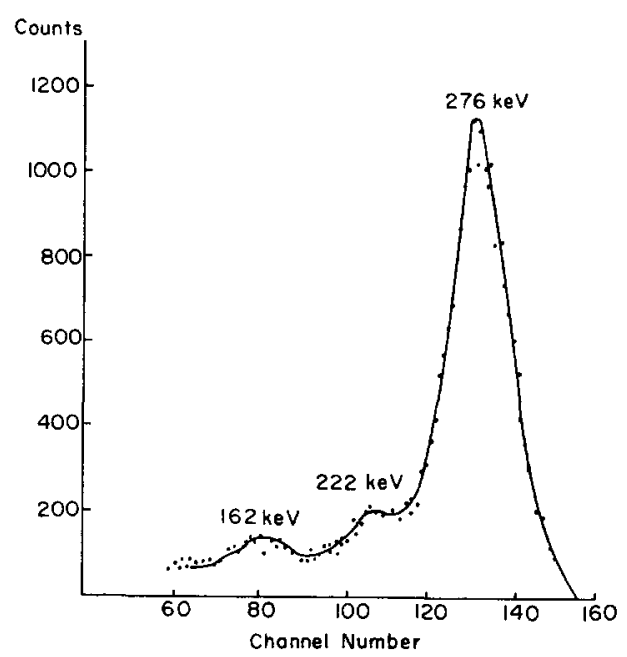

Fig. 4. Spectrum of gamma rays in coincidence with the energy range 148 to $172 \mathrm{keV}$.

\section{Directional Correlation Results}

Since the $82 \mathrm{keV}$ level has a half-life ${ }^{7}$ ) of $6.31 \pm 0.05 \mathrm{~ns}$, possible attenuation of the directional correlations involving this level as an intermediate state may occur. Several authors have investigated this possibility. Clikeman and Stewart ${ }^{9}$ ) used both solid $\mathrm{BaCl}_{2}$ and liquid sources for the correlation of the $356 \mathrm{keV}-82 \mathrm{keV}$ cascade. They found no difference in anisotropy within experimental error. Bodenstedt et al. ${ }^{7}$ ) used a liquid source to measure the correlation of the same cascade, and observed the anisotropy as a function of the time delay between the two gamma rays. Their results also showed no observable attenuation. Based on the conclusion reached by these authors, as well as the fact that the source used in the present experiment is in the form of dilute $\mathrm{HCl}$ solution, no perturbation of the directional correlations involving the $82 \mathrm{keV}$ level as the intermediate state is expected. Since the half-life 
of the $162 \mathrm{keV}$ level is shorter ( $\leqq 0.5 \mathrm{~ns}$, see ref. $\left.{ }^{7}\right)$ ) than that of the $82 \mathrm{keV}$ level, it is also assumed that no corrections for the perturbations by extranuclear fields are necessary for the $276 \mathrm{keV}-162 \mathrm{keV}$ directional correlation function.

\subsection{THE $356 \mathrm{keV}-82 \mathrm{keV}$ CASCADE}

One differential analyser was set to accept the $356 \mathrm{keV}$ photopeak, ("A" of fig. 5), being careful to avoid contribution of the $302 \mathrm{keV}$ line; while the other analyser accepted the photopeak of the $82 \mathrm{keV}$ gamma ray. About $2.7 \times 10^{5}$ true coincidences were accumulated at each angle. The normalized expansion coefficients, after correction for finite resolution, are:

$$
A_{2}=0.0331 \pm 0.0017, \quad A_{4}=0.0045 \pm 0.0033 \text {. }
$$

Although the error in $A_{4}$ is large enough to cast doubt upon its sign, it is sufficiently small to restrict the magnitude of the absolute value of $A_{4}$.

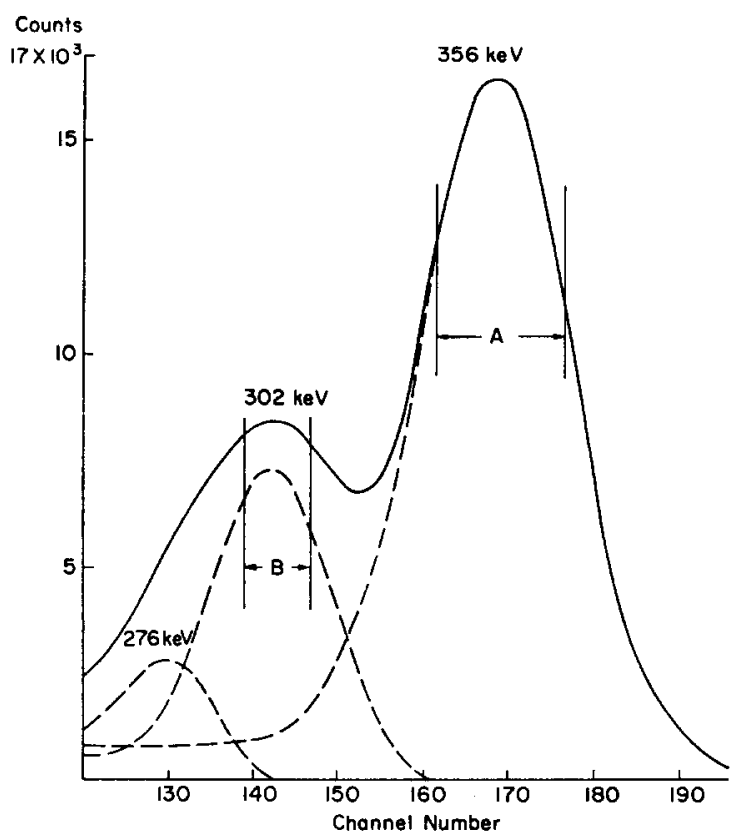

Fig. 5. Spectrum of gamma rays in coincidence with the energy range 74 to $94 \mathrm{keV}$. The complex peaks are resolved with the mono-energetic pulse height shapes of $\mathbf{H g}^{208}$ (279 $\mathrm{keV}$ ) and $\mathrm{Sn}^{118}(392$ keV).

\subsection{THE $302 \mathrm{keV}-82 \mathrm{keV}$ CASCADE}

Since interference from other cascades, notably that of $356 \mathrm{keV}-82 \mathrm{keV}$, was unavoidable, the spectrum of gamma rays in coincidence with $82 \mathrm{keV}$ was taken. The photopeaks at 356,302 and $276 \mathrm{keV}$ in this spectrum were resolved with the help of the mono-energetic pulse height shapes of $\mathrm{Hg}^{203}$ (279 keV) and $\mathrm{Sn}^{113}$ (392 
$\mathrm{keV}$ ), as shown in fig. 5. The differential analyser accepting the $302 \mathrm{keV}$ gamma ray was set at position " $B$ " of fig. 5, with a negligible amount of overlap with the 276 $\mathrm{keV}$ line. The other discriminator was set to accept the $82 \mathrm{keV}$ photopeak. The interference from the $356 \mathrm{keV}-82 \mathrm{keV}$ cascade was found to be $14 \%$.

A total of about $7.3 \times 10^{4}$ true coincidences were accumulated at each angle, and the normalized expansion coefficients, after correcting for finite resolution, are:

$$
A_{2}=-0.0159 \pm 0.0052, \quad A_{4}=0.0059 \pm 0.0077 \text {. }
$$

The revised coefficients, after correction for the interference of the $356 \mathrm{keV}-82 \mathrm{keV}$ cascade, are:

$$
A_{2}=-0.0238 \pm 0.0060, \quad A_{4}=0.0061 \pm 0.0089
$$

\subsection{THE $80 \mathrm{keV}-82 \mathrm{keV}$ CASCADE}

For this cascade, each discriminator was set to accept both of the photopeaks. Using the method described in subsect. 4.2, the interference from the $356 \mathrm{keV}-82 \mathrm{keV}$ cascade was found to be $10 \%$, and that of the $302 \mathrm{keV}-82 \mathrm{keV}$ cascade, $3 \%$. The true coincidences accumulated at each angle were about $4.7 \times 10^{4}$, and the final normalized coefficients, corrected for both angular resolution and interferences, are:

$$
A_{2}=0.0319 \pm 0.0045, \quad A_{4}=0.005 \pm 0.009 \text {. }
$$

\subsection{THE $276 \mathrm{keV}-162 \mathrm{keV}$ CASCADE}

Although the coincidence rate for this cascade is quite small compared to the other cascades, the interferences from other cascades are not present; and the anisotropy is considerably more pronounced than the others. The differential analysers were set to accept the energy ranges of 140 to $180 \mathrm{keV}$ and 254 to $294 \mathrm{keV}$ respectively. A total of about $2 \times 10^{4}$ true coincidences were accumulated at each angle, and the normalized coefficients, after geometry corrections, are:

$$
A_{2}=-0.328 \pm 0.009, \quad A_{4}=-0.067 \pm 0.016 \text {. }
$$

\section{Interpretation and Discussion}

\subsection{THE $356 \mathrm{keV}-82 \mathrm{keV}$ CASCADE}

The spins of the ground state of $\mathrm{Cs}^{133}$ and $\mathrm{Ba}^{133}$ are $\left.{ }^{10,17}\right) \frac{7}{2}^{+}$and $\frac{1}{2}^{+}$respectively, in agreement with shell model predictions of $g_{\frac{2}{2}}$ and $s_{\frac{1}{2}}$. Judging from the allowed nature of the electron capture transition from the ground state of $\mathrm{Ba}^{133}\left(\frac{1}{2}{ }^{+}\right)$to the $438 \mathrm{keV}$ level, $\left.\left(\log f t=5.6^{14}\right) ; \log f t=6.1^{12}\right)$ ), and the fact that the $438 \mathrm{keV}$ level has not been reached by Coulomb excitation ${ }^{5}$ ), the spin and parity of the $438 \mathrm{keV}$ level is assumed to be $\frac{1}{2}^{+}$. The $82 \mathrm{keV}$ level, on the other hand, has been reached by Coulomb excitation ${ }^{5}$ ). It is populated from the ground state ${ }^{14}$ ) of $\mathrm{Xe}^{133}\left(\frac{3}{2}{ }^{+}\right)$ through an allowed $\beta$ transition $\left(\log f t=5.6\right.$ see ref. $\left.{ }^{14}\right)$ ); but not populated by an electron capture transition from the ground state of $\mathrm{Ba}^{133}\left(\frac{1}{2}^{+}\right)$. All these facts, in 
addition to the predominantly M1 nature of the $82 \mathrm{keV}$ gamma ray ${ }^{9}$ ) as deduced from its internal conversion coefficient, combine to assign uniquely a spin and parity of $\frac{5}{2}^{+}$to the $82 \mathrm{keV}$ level.

The spin sequence for the $356 \mathrm{keV}-82 \mathrm{keV}$ cascade is therefore $\frac{1}{2}^{+}(Q) \frac{5}{2}^{+}(D, Q)^{\frac{7}{2}}{ }^{+}$. The theoretical $A_{2}^{(1)}$ and $A_{4}^{(1)}$ obtained from the table of $F$ coefficients ${ }^{18}$ ) are:

$$
A_{2}^{(1)}=-0.5345, \quad A_{4}^{(1)}=-0.6172 \text {. }
$$

Knowing $A_{2}=0.0331 \pm 0.0017$ and $A_{4}=0.0045 \pm 0.0033$, one obtains for the 82 $\mathrm{keV}$ gamma ray:

$$
A_{2}^{(2) \exp }=-0.0619 \pm 0.0032, \quad A_{4}^{(2) \exp }=-0.0073 \pm 0.0053
$$

Using the method developed by Arns and Wiedenbeck ${ }^{19}$ ), as shown in fig. 6, it is seen that the two solutions of $Q$, the quadrupole content of the $82 \mathrm{keV}$ gamma ray, are:

$$
Q_{1}=0.024 \pm 0.001, \quad Q_{2}=0.924 \pm 0.002
$$

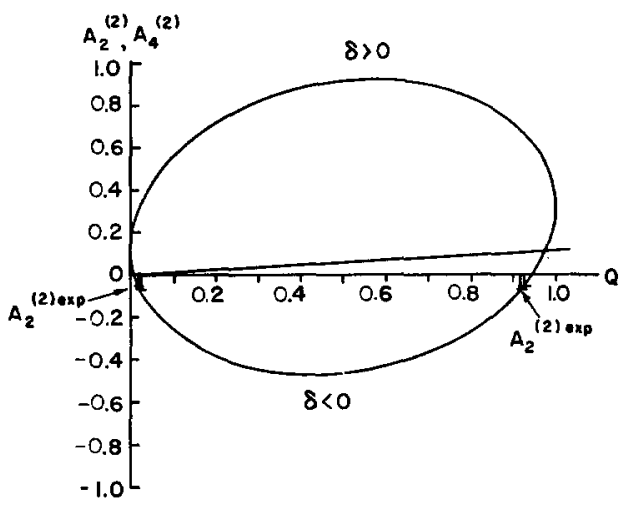

Fig. 6. $A_{2}{ }^{(2)}$ and $A_{4}{ }^{(2)}$ versus $Q$, the quadrupole content, for the transition $\frac{5}{2} \rightarrow \frac{7}{2}$.

The magnitude of $A_{4}^{(2) \text { exp }}$, although negative, requires one to choose $Q_{1}$ as the correct solution. This quadrupole mixture is also in agreement with the predominantly M1 nature of the $82 \mathrm{keV}$ gamma ray deduced from its internal conversion coefficient ${ }^{9}$ ) $\alpha=1.77 \pm 0.05$. Thus once can conclude that the mixing ratio of the $82 \mathrm{keV}$ gamma ray is $97.6 \pm 0.1 \% \mathrm{M} 1$. and $2.4 \pm 0.1 \% \mathrm{E} 2$.

Using this mixing ratio and the value ${ }^{7}$ ) $6.31 \pm 0.05 \mathrm{~ns}$ as the half-life of the 82 $\mathrm{keV}$ level, calculations analogous to those of Clikeman and Stewart ${ }^{9}$ ) show that the $82 \mathrm{keV}$ transition is indeed single-particle in character. While its E2 transition rate is enhanced by only a factor $\sim 2$ from theoretical single-particle estimates ${ }^{20}$ ), its M1 rate is retarded by a factor $\sim 400$. Such a retardation supports the interpretation of the $82 \mathrm{keV}$ level as $\mathrm{d}_{\frac{\mathbf{s}}{2}}$, thus making the $82 \mathrm{keV}$ transition essentially $l$-forbidden $\left(\mathrm{d}_{\frac{\pi}{2}}-\mathrm{g}_{7} ; \Delta l=2\right)$. 


\subsection{THE $302 \mathrm{keV-82} \mathrm{keV}$ CASCADE}

The $384 \mathrm{keV}$ level is assigned as the $\mathrm{d}_{3}$ single-particle state by all recent authors ${ }^{7-9,12}$ ). It has been reached by Coulomb excitation ${ }^{5}$ ), and is populated by an electron capture transition from the ground state of $\mathrm{Ba}^{133}\left(\mathrm{~s}_{\frac{1}{2}}\right)$ with a $\log f t$ value $^{12}$ ) of 7.2. This high value of $\log f t$ for an allowed transition suggests $l$-forbiddenness ${ }^{8,12}$ ), and supports the assignment of $\mathrm{d}_{\frac{3}{2}}$ to the $384 \mathrm{keV}$ level. The 302 keV-82 keV cascade, therefore, has the spin sequence $\frac{3}{2}^{+}(D, Q) \frac{5}{2}^{+}(D, Q) \frac{7}{2}^{+}$. Knowing $A_{2}=-0.0238 \pm 0.0060$ and $A_{4}=0.0061 \pm 0.0089$, and using $A_{2}^{(2)}$ and $A_{4}^{(2)}$ for the $82 \mathrm{keV}$ gamma ray obtained from the $356 \mathrm{keV}-82 \mathrm{keV}$ correlation, $A_{2}^{(1) \exp }$ and $A_{4}^{(1) \text { exp }}$ for the $302 \mathrm{keV}$ gamma ray are found to be:

$$
A_{2}^{(1) \exp }=0.384 \pm 0.098, \quad A_{4}^{(1) \exp }=-0.8 \pm 1.3 .
$$

As shown in fig. $7, A_{2}^{(1) \exp }$ gives the following two solutions for $Q$ :

$$
Q_{1}=0.003 \pm 0.001, \quad Q_{2}=0.914 \pm 0.029 \text {. }
$$

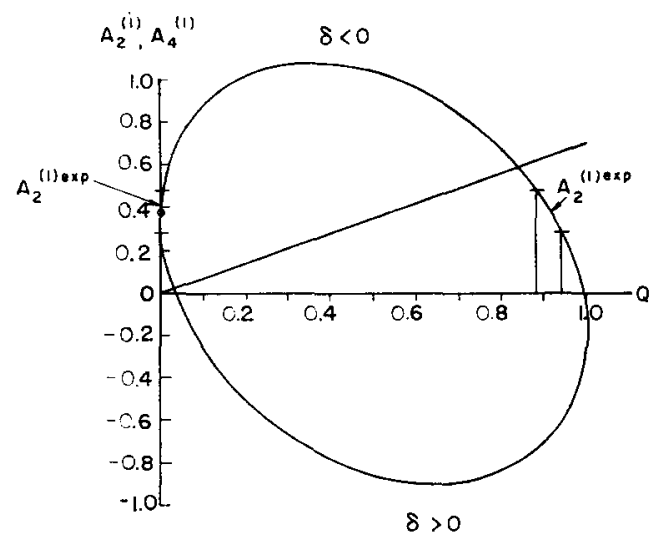

Fig. 7. $A_{2}{ }^{(1)}$ and $A_{4}{ }^{(1)}$ versus $Q$ for the transition $\frac{3}{2} \rightarrow \frac{5}{2}$.

It is obvious that due to the large error in $A_{4}^{(1) \exp }$, it cannot serve to help the choice of $Q$ in this case. Neither is the measured internal conversion coefficient for the 302 $\mathrm{keV}$ transition known with sufficient accuracy ${ }^{14}$ ) to facilitate the choice. However, judging from the single-particle interpretation of the 384 and the $82 \mathrm{keV}$ levels, the $302 \mathrm{keV}$ gamma ray should be predominantly Ml in character. It is then concluded that the mixing ratio of the $302 \mathrm{keV}$ gamma ray is $99.7 \pm 0.1 \% \mathrm{M} 1$ and $0.3 \pm 0.1 \% \mathrm{E} 2$.

\subsection{THE $80 \mathrm{keV}-82 \mathrm{keV}$ CASCADE}

With $A_{2}=0.0319 \pm 0.0045$ and $A_{4}=0.005 \pm 0.009$, and using $A_{2}^{(2)}$ and $A_{4}^{(2)}$ for the $82 \mathrm{keV}$ gamma ray from the $356 \mathrm{keV}-82 \mathrm{keV}$ correlation results, $A_{2}^{(1) \exp }$ and $A_{4}^{(1) \exp }$ for the $80 \mathrm{keV}$ gamma ray are calculated to be:

$$
A_{2}^{(1) \exp }=-0.515 \pm 0.077, \quad A_{4}^{(1) \exp }=-0.7 \pm 1.3 \text {. }
$$


Due to the error in $A_{4}^{(1) \text { exp }}$, this result could fit either a $\frac{3}{2}(D, Q) \frac{5}{2}(D, Q) \frac{7}{2}$ sequence or a $\frac{5}{2}(D, Q) \frac{5}{2}(D, Q) \frac{7}{2}$ sequence. However, as will be seen in subsect. 5.4, the 276 $\mathrm{keV}-162 \mathrm{keV}$ directional correlation measurement has unambiguously determined the spin of the $162 \mathrm{keV}$ level to be $\frac{5}{2}$. Therefore, the former sequence is eliminated.

From fig. 8, one obtains the two solutions of $Q$ for the $80 \mathrm{keV}$ gamma ray as:

$$
Q_{1}=0.019 \pm 0.019, \quad Q_{2}=0.63 \pm 0.09 \text {. }
$$

It will also be shown in subsect. 5.4 that the $162 \mathrm{keV}$ level can no longer be characterized as a single-particle level due to the predominantly E2 character of the 162 $\mathrm{keV}$ gamma ray. In view of this result, one would also expect an enhancement of the quadrupole content of the $80 \mathrm{keV}$ gamma ray; and, therefore select $Q_{2}$ as the likely solution.

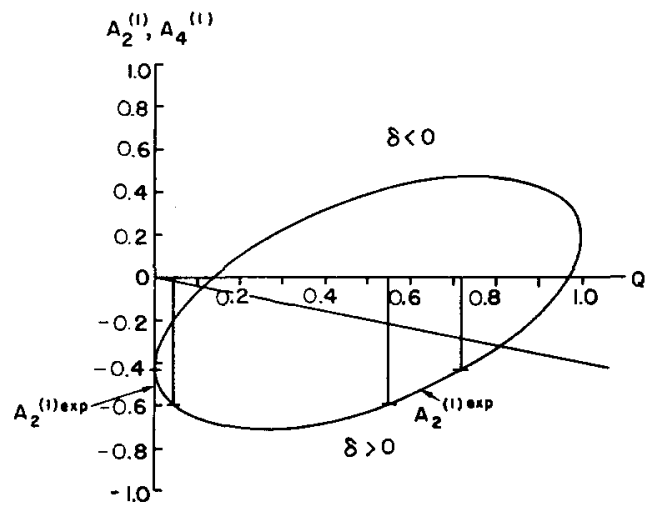

Fig. 8. $A_{2}^{(1)}$ and $A_{4}^{(1)}$ versus $Q$ for the transition $\frac{6}{2} \rightarrow \frac{5}{2}$.

\subsection{THE $276 \mathrm{keV}-162 \mathrm{keV}$ CASCADE}

The experimental coefficients for this cascade are:

$$
A_{2}=-0.328 \pm 0.009, \quad A_{4}=-0.067 \pm 0.016 \text {. }
$$

Since the spin of the $438 \mathrm{keV}$ level is $\frac{1}{2}$, and that of the ground state, $\frac{7}{2}$, both $\frac{3}{2}$ and $\frac{5}{2}$ are possible values for the intermediate $162 \mathrm{keV}$ level. Therefore, the $276 \mathrm{keV}-162 \mathrm{keV}$ cascade could have either the $\frac{1}{2}(D, Q) \frac{3}{2}(Q) \frac{7}{2}$ or the $\frac{1}{2}(Q) \frac{5}{2}(D, Q) \frac{7}{2}$ sequence.

Assuming $\frac{1}{2}(D, Q) \frac{3}{2}(Q) \frac{7}{2}$ is the correct sequence, from the table of $F$ coefficients ${ }^{18}$ ), one obtains:

$$
A_{2}^{(2)}=-0.1428, \quad A_{4}^{(2)}=0 .
$$

The fact that $A_{4}^{(2)}$ is zero requires $A_{4}$ to be also zero. The experimental $A_{4}$ clearly does not satisfy this requirement. Furthermore, the value of $A_{2}^{(1) e x p}$ computed from $A_{2}$ and $A_{2}^{(2)}$ is $2.297 \pm 0.063$. Since in the $A_{2}^{(1)}$ versus $Q$ graph of the transition ${ }^{19}$ ) $\frac{1}{2} \rightarrow \frac{3}{2}$ the highest point of the ellipse has a value of $A_{2}^{(1)}=1.0$, the $A_{2}^{(1) \text { exp }}$ obtained 
above cannot possibly fit this spin sequence. Therefore, one is obliged to discard $\frac{3}{2}$ as a possible value for the spin of the $162 \mathrm{keV}$ level.

In the $\frac{1}{2}(Q) \frac{5}{2}(D, Q) \frac{7}{2}$ sequence, the $F$ coefficients give:

Consequently,

$$
A_{2}^{(1)}=-0.5345, \quad A_{4}^{(1)}=-0.6172 .
$$

$$
A_{2}^{(2) \exp }=0.614 \pm 0.017, \quad A_{4}^{(2) \exp }=0.108 \pm 0.026 .
$$

Fig. 9 shows that the two values of $Q$ required by $A_{2}^{(2) e x p}$ are:

$$
Q_{1}=0.123 \pm 0.009, \quad Q_{2}=0.951 \pm 0.006 \text {. }
$$

Since the value of $A_{4}^{(2) e x p}$ restricts $Q$ to be greater than 0.695 , the mixing ratio of the $162 \mathrm{keV}$ gamma ray is therefore $95.1 \pm 0.6 \% \mathrm{E} 2$ and $4.9 \pm 0.6 \% \mathrm{M} 1$.

The predominantly E2 character of the $162 \mathrm{keV}$ gamma ray indicates that singleparticle interpretation can no longer be applied to the $162 \mathrm{keV}$ level.

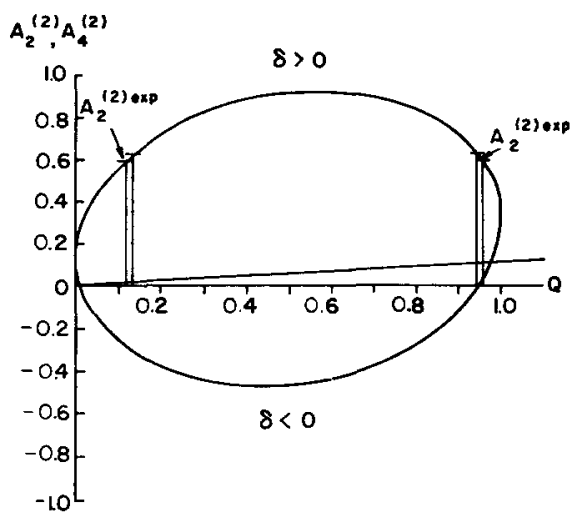

Fig. 9. $A_{2}^{(2)}$ and $A_{4}^{(2)}$ versus $Q$ for the transition $\frac{1}{2} \rightarrow$

\section{Summary and Conclusion}

The present investigation confirms the decay scheme as shown in fig. 1. The measurements also give support to the predominantly single-particle character of the ground state, 82,384 and $438 \mathrm{keV}$ levels of $\mathrm{Cs}^{133}$ as $\mathrm{g}_{\frac{7}{2}}, \mathrm{~d}_{\vec{k}}, \mathrm{~d}_{\frac{1}{3}}$, and $\mathrm{s}_{\frac{1}{2}}$ respectively. The spin of the $162 \mathrm{keV}$ level has been unambiguously determined to be $\frac{5}{2}$. Since this level has been reached by Coulomb excitation ${ }^{5}$ ), its parity is therefore + . If an electron capture transition to the $162 \mathrm{keV}$ level did exist, as deduced by Stewart and Lu ${ }^{8}$ ), then the assignment of $\frac{5}{2}{ }^{+}$to the $162 \mathrm{keV}$ level would require such a transition to be second-forbidden rather than allowed. Judging from the magnitude of the log $f t$ value $\left.{ }^{8,14}\right)(\log f t=9.0)$ such a requirement does not seem unreasonable. In contrast to the other levels, the character of the $162 \mathrm{keV}$ level cannot be interpreted from the single-particle point of view. 
The mixing ratios of the following gamma rays are determined to be: $80 \mathrm{keV}$ : $(63 \pm 9) \% \mathrm{E} 2+(37 \pm 9) \% \mathrm{M} 1 ; 82 \mathrm{keV}:(97.6 \pm 0.1) \% \mathrm{M} 1+(2.4 \pm 0.1) \% \mathrm{E} 2 ; 162 \mathrm{keV}:$ $(95.1 \pm 0.6) \% \mathrm{E} 2+(4.9 \pm 0.6) \% \mathrm{M} 1 ; 276 \mathrm{keV}:$ pure E2; $302 \mathrm{keV}:(99.7 \pm 0.1) \%$ $\mathrm{M} 1+(0.3 \pm 0.1) \% \mathrm{E} 2$; and $356 \mathrm{keV}$ : pure E2.

Note: During the last stages of this work, an article was published by Münnich, Fricke and Wellner ${ }^{21}$ ) on the directional correlation measurements in the decay of $\mathrm{Ba}^{133}$. The results of the present work, especially with regard to the spin determination of the $162 \mathrm{keV}$ level, agree quite well with the results of these authors.

\section{References}

1) R. W. Hayward, D. D. Hoppes and H. Ernst, Phys. Rev. 93 (1954) 916A

2) M. Langevin, Compt. Rend. 238 (1954) 1310; 240 (1955) 289; Ann. Phys. 1 (1956) 57

3) B. Crasemann, J. G. Pengra and I. E. Lindstrom; Phys. Rev. 108 (1957) 1500

4) R. K. Gupta, S. Jha, M. C. Joshi and B. K. Madan, Nuovo Cim. 8 (1958) 48

5) L. W. Fagg, Phys. Rev. 109 (1958) 100

6) S. D. Koicki, A. M. Mijatovic and J. M. Simic, Bull. Inst. Nuc. Sci. Boris Kidrich (Belgrade) 8 (1958) 1

7) E. Bodenstedt, H. J. Körner and E. Matthias, Nuclear Physics 11 (1959) 584

8) M. G. Stewart and D. C. Lu, Phys. Rev. 117 (1960) 1044

9) F. M. Clikeman and M. G. Stewart, Phys. Rev. 117 (1960) 1052

10) J. E. Mack, Revs. Mod. Phys. 22 (1950) 64

11) M. K. Ramaswamy, W. L. Skeel and P. S. Jastram, Nuclear Physics 16 (1960) 619

12) M. K. Ramaswamy, W. L. Skeel and P. S. Jastram, Nuclear Physics 19 (1960) 299

13) A. P. Arya, Phys. Rev. 122 (1961) 549

14) Nuc. Data Sheets, Nat. Acad. Sci., Nat. Research Council: Washington, D. C. ,NRC61-2-84

15) M. E. Rose, Phys. Rev. 91 (1953) 610

16) Arns, Sund and Wiedenbeck, Univ. of Mich. Research Institute, Report 2375-4-T (Feb. 1959)

17) M. Goldhaber and R. D. Hill, Revs. Mod. Phys. 24 (1952) 217

18) M. Ferentz and N. Rosenzweig, Table of $F$ coefficients, ANL-5324 (1955)

19) R. G. Arns and M. L. Wiedenbeck, Phys. Rev. 111 (1958) 1631

20) S. A. Moszkowski in Beta- and gamma-ray spectroscopy, ed. by K. Siegbahn (North-Holland Publishing Co. Amsterdam, 1955) chapt. 13

21) F. Münnich, K. Fricke and U. Wellner, Z. f. Phys. 174 (1963) 68 